\title{
Physician-Related Barriers to Hypertension Management
}

\author{
Lexin Wang \\ School of Biomedical Sciences, Charles Sturt University, Wagga Wagga, Australia
}

\section{Key Words}

Hypertension management barriers · WHO-ISH guidelines

\begin{abstract}
Objective: To investigate the levels of understanding and implementation of current hypertension guidelines among specialist physicians. Subjects and Methods: A survey of the understanding and practice in the management of hypertension was conducted among 56 physicians who individually had managed more than 150 new hypertensive patients annually. Results: All respondents were familiar with the current hypertension guidelines and $51.8 \%$ always or usually followed these guidelines in treating their patients. Most believed that the lowest blood pressure where pharmacological therapy should be initiated was $155 / 95 \mathrm{~mm}$ Hg. Forty-five (80.4\%) physicians considered a blood pressure of less than 140/ $90 \mathrm{~mm} \mathrm{Hg}$ should be achieved in hypertensive patients. Only $12(21.4 \%)$ respondents provided routine advice on lifestyle modification to their patients. Fifty-four (96.4\%) respondents ranked poor adherence to antihypertensive drugs as the major patient barrier to blood pressure control. Conclusions: Most physicians are familiar with the current hypertension guidelines but the implementation of these guidelines in clinical practice is inadequate. Systematic educational programs are needed to enhance physicians' awareness of the optimal goals of blood pressure control.
\end{abstract}

Copyright $@ 2004$ S. Karger AG, Basel

\section{Introduction}

Although improving the quality of hypertension care is a priority, most patients with hypertension have inadequate control of blood pressure [1-6], leading to increased risk of coronary artery disease, congestive heart failure, renal insufficiency and/or stroke [1]. Currently, safe and effective antihypertensive therapies are readily available to most hypertensive patients, therefore it is of paramount importance to identify the barriers to obtaining optimal blood pressure control in order to maximize the therapeutic effects and to reduce the morbidity and mortality associated with hypertension.

Previous studies on poor control of blood pressure have mainly focused on patient compliance with antihypertensive therapies and patients' characteristics associated with non-compliance $[1,7]$. Recent data have shown that physicians may not be aggressive enough in the management of hypertension [8-10]. Therefore the primary purpose of the study was to evaluate physicians' attitude towards the target level of blood pressure in hypertensive patients. Physicians' practice in hypertension management and their perceptions of the barriers to blood pressure control were also assessed in the study.
Lexin Wang, MD, PhD

School of Biomedical Sciences

Charles Sturt University

Wagga Wagga, NSW 2678 (Australia)

Tel. +61 26933 2905, Fax +6126933 2587, E-Mail lwang@csu.edu.au 


\section{Subjects and Methods}

\section{Subjects}

From November 2001 to December 2002, 56 cardiologists were interviewed on their knowledge of the management of hypertension and perceptions of the barriers to blood pressure control. These physicians were randomly selected from 12 regional hospitals in southeast China and informed consent was obtained from all of them prior to conducting the survey.

Each physician had more than 12 years of experience in medical practice and had been a cardiologist for more than 4 years at the time of the survey. Each physician had managed more than 150 new hypertensive patients per year in the last 3 years before the survey.

\section{Survey Questionnaires}

The questions covered by the interview included: (1) awareness of the 1999 World Health Organization-International Society of Hypertension (WHO-ISH) guidelines for hypertension management [11], which were widely used as the local guidelines in China. The key aspects of the guideline were to initiate antihypertensive therapy in patients with grade I and II hypertension once the concurrent risk factors have been taken into consideration. The recommended antihypertensive drugs in these guidelines are diuretics, beta-blockers, angiotensin-converting enzyme (ACE) inhibitors, calcium antagonists, alpha-blockers and $\mathrm{AT}_{1}$-receptor blockers, with diuretics and beta-blockers as the first-line drugs; (2) the lowest blood pressure at which pharmacological therapy should be started, if there were no other concurrent risk factors; (3) the target blood pressure for hypertension treatment; (4) preferred first-line antihypertensive drug; (5) awareness of uncontrolled hypertension; (6) recommendation of lifestyle modification; (7) perceptions on patients' barriers to blood pressure control.

During the interview all the cardiologists were encouraged to freely express their views on the practice and quality of hypertension management among their patients. Since this study was a descriptive survey, no statistical analysis was attempted.

\section{Results}

\section{Awareness of Hypertension Guidelines}

All of the 56 physicians who were approached to take part in the study agreed to the request and completed the survey. They were aware of the new WHO-ISH guidelines. More than half $(51.8 \%)$ of the physicians indicated that they always or usually follow the WHO-ISH guidelines when treating patients (table 1 ).

The lowest systolic and diastolic blood pressure at which pharmacological treatment should be started is shown in table 1 . On average, the physicians reported that 155/95 mm Hg was the lowest systolic and diastolic blood pressure at which they would recommend pharmacologic treatment to patients.

Forty-five $(80.4 \%)$ physicians considered the target blood pressure should be less than 140/90 $\mathrm{mm} \mathrm{Hg}$ during pharmacological therapy as recommended by WHO-ISH,
Table 1. Physician's awareness and implementation of hypertension guidelines

\begin{tabular}{|c|c|}
\hline Index & Values \\
\hline \multicolumn{2}{|l|}{ Familiar with WHO-ISH guidelines? } \\
\hline Yes, very familiar & $38(67.9 \%)$ \\
\hline Yes, somewhat familiar & $18(32.1 \%)$ \\
\hline No & 0 \\
\hline \multicolumn{2}{|c|}{ Agree with WHO-ISH treatment recommendations? } \\
\hline Yes & $45(80.4 \%)$ \\
\hline No & $11(19.6 \%)$ \\
\hline \multicolumn{2}{|c|}{$\begin{array}{l}\text { Follow WHO-ISH treatment recommendations } \\
\text { when treating patients? }\end{array}$} \\
\hline Always or usually & $29(51.8 \%)$ \\
\hline Sometimes & $27(48.2 \%)$ \\
\hline Never/rarely & 0 \\
\hline \multicolumn{2}{|c|}{$\begin{array}{l}\text { Lowest SBP at which you would recommend } \\
\text { drug therapy to a patient }{ }^{1}\end{array}$} \\
\hline With co-morbidities, $\mathrm{mm} \mathrm{Hg}$ & $153.2 \pm 8.4$ \\
\hline Without co-morbidities, $\mathrm{mm} \mathrm{Hg}$ & $158.6 \pm 7.7$ \\
\hline \multicolumn{2}{|c|}{$\begin{array}{l}\text { Lowest DBP at which you would recommend } \\
\text { drug therapy to a patient }{ }^{1}\end{array}$} \\
\hline With co-morbidities, $\mathrm{mm} \mathrm{Hg}$ & $93.6 \pm 2.5$ \\
\hline Without co-morbidities, $\mathrm{mm} \mathrm{Hg}$ & $98.3 \pm 4.7$ \\
\hline $\begin{array}{l}\mathrm{SBP}=\text { Systolic blood pressure; } \\
\text { Results are expressed as means }\end{array}$ & od pressure. \\
\hline
\end{tabular}

while 11 (19.6\%) believed 160/95 mm Hg was more practical.

\section{Use of Antihypertensive Drugs}

Thirty-two (57.1\%) preferred calcium channel blockers as the first-line therapy for hypertension, provided there was no contraindication, no co-morbidity (e.g. stroke or a previous myocardial infarction) and no financial constraints from patients. Twenty $(35.7 \%)$ preferred ACE inhibitors as the first-line treatment, and the remaining 4 (7.1\%) would initiate anti-hypertensive therapy with diuretics. When specifically asked the most appropriate first-step drug for patients who were more than 60 years old, the preference of calcium channels blockers, ACE inhibitors and diuretics remained unchanged.

All of the physicians indicated that the combination of two or more antihypertensive drugs was necessary if monotherapy had failed to control blood pressure below the target level.

When asked the percentage of treated hypertensive patients who have their blood pressure under control, 52 $(92.8 \%)$ reported $60-70 \%$ of the patients treated with anti-hypertensive medications had controlled blood pressure. 


\section{Non-Pharmacological Treatment}

Forty-two (75.0\%) believed lifestyle modification should facilitate pharmacotherapy of hypertension, but only $12(21.4 \%)$ routinely provided advice on dietary modification (low salt and potassium-rich diet), regular physical exercises and weight reduction.

\section{Perceived Patient Barriers to Blood Pressure Control}

Fifty-four $(96.4 \%)$ respondents ranked poor adherence to antihypertensive drugs as the major patient barrier to blood pressure control. Of these, $50(92.6 \%)$ attributed poor medication adherence to patients' financial constraints, whereas $4(7.4 \%)$ ranked adverse effects as the leading cause of poor drug compliance. Thirty-four $(60.7 \%)$ reported at least one of their patients had missed a hospital appointment in the week before the survey.

\section{Discussion}

Although there have been significant advances in the understanding of pharmacological therapy of hypertension in the last three decades, only $25 \%$ of patients being treated for hypertension have blood pressure values less than $140 / 90 \mathrm{~mm} \mathrm{Hg}$ [1-5]. In China, less than $5 \%$ of treated hypertensive patients have blood pressure below 140/90 mm Hg [6]. The uncontrolled blood pressure has significant adverse impact on cardiovascular complications and, thus, on healthcare costs.

The suboptimal control of blood pressure is related to a number of factors, including failure to diagnose high blood pressure in clinics and hospitals, inadequate treatment regimens, side effects of antihypertensive medications, poor patient adherence to medications, and lifestyle choices [7, 10-13].

The present descriptive survey shows that, although most physicians were familiar with the new hypertension guidelines, nearly $20 \%$ of them did not agree with the WHO-ISH recommendations, and only a little more than half of the respondents closely follow these guidelines when treating hypertensive patients. More importantly, most respondents believed that the lowest blood pressure for initiation of pharmacological therapy is $155 / 95 \mathrm{~mm}$ $\mathrm{Hg}$, which is substantially above the recommendation of current guidelines [11]. Furthermore, about $20 \%$ of our respondents set a target blood pressure of $160 / 95 \mathrm{~mm} \mathrm{Hg}$. These results suggest that many physicians were not aggressive enough in treating hypertension.

Lifestyle modification, such as low salt and high potassium diet, regular exercise and weight reduction, facili- tates pharmacological control of blood pressure, and should be recommended to all patients whenever appropriate [11]. Lifestyle modification has been one of the most frequently cited impediments to blood pressure control [14]. In the present study, only $21.4 \%$ of the physicians provided regular advice or consultation on lifestyle changes to their patients. These data indicate that there is a need for substantial improvement in this important therapeutic area.

Patients' adherence to pharmacotherapy has been identified as one of the main reasons for uncontrolled hypertension [1, 2, 11]. Multiple-dose regimens reduce patients' adherence to antihypertensive medications [11, 15, 16]. Consistent with previous findings, most of our respondents reported poor adherence to antihypertensive drugs as the leading patient-related barrier to blood pressure control. The costs of medication and hospital visits have been identified as a major contributing factor for poor patient adherence to pharmacological treatment. Among the seven classes of antihypertensive drugs recommended in the 1999 WHO-ISH guidelines, diuretics are the cheapest whereas the $\mathrm{AT}_{1}$-receptor blockers are the most expensive in China. The ALLHAT trial indicated that thiazide-type diuretics are superior to doxazosin, an alpha-blocker, in preventing one or more major forms of cardiovascular disease [17]. In spite of the low cost and clearly defined clinical efficacy of diuretics, it was not the first-line drug of the physicians who participated in the survey, as only $7.1 \%$ of them would initiate drug therapy with a diuretic.

The present study has found that most cardiologists are aware of the recommendations of new hypertension guidelines. However, the implementation of these guidelines in clinical practice is variable. Many physicians start pharmacological therapy at a blood pressure level higher than the current recommendation of $140 / 90 \mathrm{~mm} \mathrm{Hg}$. The target blood pressure during therapy is also higher than that recommended by the hypertension guidelines. In addition, only a small proportion of physicians routinely provide advice on lifestyle modification to their patients.

\section{Conclusion}

The results of this survey indicate that there is an urgent need to establish nationwide educational and quality monitoring programs to facilitate the implementation of new hypertension guidelines in clinical practices in China. 


\section{References}

1 Joint National Committee on Prevention, Detection, Evaluation, and Treatment of High Blood Pressure. The Sixth Report of the Joint National Committee on Prevention, Detection, Evaluation and Treatment of High Blood Pressure (JNC VI). Arch Intern Med 1997;157: 2413-2446.

2 Berlowitz, DR, Ash AS, Hickey EC, Friedman RH, Glickman M, Quadra B, Moskowitz MA: Inadequate management of blood pressure in a hypertensive population. N Engl J Med 1998; 339:1957-1963.

3 Mancia G, Sega R, Milesi C, Cesana G, Zanchetti A: Blood-pressure control in the hypertensive population. Lancet 1997;349:454-457.

4 Fagard RH, Van den Enden M, Leeman M, Warling X: Survey on treatment of hypertension and implementation of World Health Organization/International Society of Hypertension risk stratification in primary care in Belgium. J Hypertens 2002;20:1297-1302.

5 Colhoun HM, Dong W, Poulter NR: Blood pressure screening, management and control in England - results from the health survey for England 1994. J Hypertens 1998;16:747-752.
6 Tao SQ, Wu XG, Duan XF, Fang WQ, Hao JS, Fan DJ, Wang WZ, Li Y: Hypertension prevalence and status of awareness, treatment and control in China. Chin Med J 1995;108:483489.

7 Anonymous: Management of patient compliance in the treatment of hypertension: Report of the NHLBI Working Group. Hypertension 1982;4:415-423.

8 Hedner T, Falk M: Physician and patient evaluation of hypertension-related risks and benefits from treatment. Blood Press 1997;6:2634.

9 Hosie J, Wiklund I: Managing hypertension in general practice: Can we do better? J Hum Hypertens 1995;9:515-518.

10 Oliveria SA, Lapuerta P, McCarthy BD, L'Italien GL, Berlowitz DR, Asch SM: Physicianrelated barriers to the effective management of uncontrolled hypertension. Arch Intern Med 2002; 162:413-423.

11 Guidelines Subcommittee. 1999 World Health Orgnization-International Society of Hypertension guidelines for the management of hypertension. J Hypertens 1999; 17:151-183.

12 Chobanian AV: Control of hypertension - an important national priority. $\mathrm{N}$ Engl J Med 2001;345:534-535.
13 Douglas JG, Ferdinand KC, Bakris GL, Sowers JR: Barriers to blood pressure control in African Americans. Overcoming obstacles is challenging, but goals can be attained. Postgrad Med 2002;112:51-56.

14 National Institutes of Health: Diagnosis and Management of Hypertension -1987: A Nationwide Survey of Physicians' Knowledge, Attitudes, and Reported Behavior. Bethesda: National Heart, Lung, and Blood Institute, US Dept of Health and Human Services, 1989. NIH publication 89-2968.

15 Eisen SA, Miller DK, Woodward RS, Spitznagel E, Przybeck TR: The effect of prescribed daily dose frequency on patient medication compliance. Arch Intern Med 1990;150:18811884.

16 Haynes RB, McKibbon KA, Kanani R: Systematic review of randomised trials of interventions to assist patients to follow prescriptions for medications. Lancet 1996;348:383386.

17 Anonymous: Major cardiovascular events in hypertensive patients randomised to doxazosin vs. chlorthalidone: The antihypertensive and lipid-lowering treatment to prevent heart attack trial (ALLHAT). ALLHAT Collaborative Research Group. JAMA 2000;283:1967-1975. 